\title{
Contenido nutricional de los alimentos promovidos por el Canal 5 de la televisión mexicana dirigidos a la población infantil
}

\author{
Nutritional content of foods \\ promoted by Channel 5 of mexican television \\ focused on child population
}

\section{ABSTRACT}

The aim of this study was to analyze the nutritional content of processed foods announced by Channel 5 of Mexican television. $T V$ programs were recorded from February 6 to 10, 2012, at the times of greatest child audience. Five independent teams recorded and validated the duration of each advertisement, product or service advertised, advertising strategy, its nutritional content and the additives they contain. 135 advertisements of unhealthy processed foods were transmitted. In contrast, none of natural and nutritious food aired. $74.0 \%$ of the time was occupied by products with high carbohydrate and sugar content $17.5 \%$ for foods high in fat and sodium; and $8.5 \%$ were dairy products with high sugar content. The results indicate that the Mexican television strongly promotes the consumption of unhealthy food to children. It is urgent that the government of Mexico to regulate the advertising of unhealthy food in children's programming schedules.

Key words: children; childhood obesity; TV food advertising; nutritional content; Mexico.

\section{INTRODUCCIÓN}

La obesidad se ha convertido en uno de los problemas de salud más graves del siglo XXI (1,2). La Organización Mundial de la Salud (OMS) estimó, que en el año 2008, existían en todo el mundo 1400 millones de personas de 20 o más años viviendo con sobrepeso, de los cuales 500 millones tenían obesidad (3). Como resultado cada año mueren 2,8 millones de personas por esa causa (4). En el año 2010, 43 millones de niños en edad preescolar (menores de 5 años) tenían sobrepeso u obesidad, lo que representa un aumento del 60 por ciento en los últimos veinte años, al incrementarse la prevalencia del $4,2 \%$ al $6,7 \%$ (5).

México se encuentra en los primeros lugares de obesidad infantil entre los países de la Organización para la Cooperación y el Desarrollo Económico $(6,7)$. De acuerdo con la última encuesta nacional probabilística de salud y nutrición, la prevalencia nacional combinada de sobrepeso y obesidad en escolares de 5-11 años de edad fue $34,4 \%$ ( $32 \%$ en mujeres y $36,9 \%$ en hombres). Estas cifras son significativamente superiores a las prevalencia encontradas en la encuesta del 2006: $26,8 \%$ en mujeres y $25,9 \%$ en hombres (8). Esta tendencia continuará aumentando si no se aplican medidas preventivas y correctivas en el campo de la salud y la nutrición.

Uno de los principales factores que contribuyen al aumento del sobrepeso y la obesidad es la existencia de "ambientes obe-
Hilda Ortiz-Pérez (1)

Nelly Molina-Frechero (1)

Ignacio Martínez-Barbabosa (1)

Rebeca Córdova-Moreno (2)

(1) Departamento de Atención a la Salud. División de Ciencias Biológicas y de la Salud. Universidad Autónoma Metropolitana Unidad Xochimilco. México. (2) Departamento de Sistemas Biológicos. División de Ciencias Biológicas y de la Salud. Universidad Autónoma Metropolitana Unidad Xochimilco. México.

Dirigir la correspondencia a:
Profesora-investigadora
Hilda Ortiz-Pérez
Licenciatura en Nutrición
Universidad Autónoma Metropolitana Unidad Xochimilco, México
Calzada del Hueso 1100,
Col. Villa Quietud, Delegación Coyoacán,
O4960 México, Distrito Federal
Tel y Fax. +52 55 5483-7202
E-mail: hortiz@Correo.xoc.uam.mx

Este trabajo fue recibido el 14 de Abril de 2015 y aceptado para ser publicado el 15 de Junio de 2015.

sogénicos", es decir, la suma de las influencias que el entorno o las condiciones de vida tienen en la promoción de la obesidad en individuos o comunidades, y que simultáneamente actúan como barreras para mantener un peso saludable $(9,10)$. Aunque se trata de un fenómeno complejo y multicausal, el tiempo dedicado a ver televisión (TDVTV) se ha asociado de manera consistente con la obesidad infantil en estudios transversales, longitudinales e intervenciones preventivas.

Desde hace más de 25 años, un estudio pionero que analizó la información de encuestas nacionales de salud en los Estados Unidos, mostró un incremento del $2 \%$ en la prevalencia de la obesidad por cada hora adicional por día dedicada ver televisión en menores de 12-17 años (11); un estudio posterior en prescolares, mostró que la hora adicional por día dedicada a ver televisión, se traducía en un aumento del $6 \%$ en la prevalencia de obesidad (12). El seguimiento de una cohorte de más de 700 niños (10-15 años) durante un período de 4 años, confirmó la fuerte relación entre el TDVTV y la prevalencia de sobrepeso al final del período, incluso después de ajustar los resultados por diversas variables (13).

En México, un análisis secundario de 18784 adolescentes (10-19 años) de la penúltima encuesta nacional de nutrición, se encontró que el TDVTV está fuertemente asociado con el sobrepeso u obesidad (14). En otro estudio de 712 menores (9-16 años), residentes en zonas de ingresos bajo y medio de la ciudad 
de México, se estimó que por cada hora diaria dedicada a ver televisión, se incrementa el riesgo de obesidad en un $12 \%$; en tanto que cada hora diaria de actividad física moderada o intensa, representa una disminución de dicho riesgo en un 10\% (15).

Para explicar la relación entre TDVTV y obesidad, diversos autores han propuesto uno o varios de los siguientes mediadores: incremento de la ingesta calórica mientras se ve la televisión; exposición a anuncios que inducen dietas de baja calidad; reducción de la actividad física; y disminución del metabolismo corporal (16-19). Un análisis de la evidencia reciente llevado a cabo por las Asociación Dietética Americana identificó los niveles de actividad física y la cantidad de TDVTV o jugar videojuegos como factores predisponentes para la obesidad (20).

Otros autores refieren que el factor más importante no es la cantidad de TDVTV, sino el número de anuncios de alimentos no saludables a que se ven expuestos los menores (21). Este tipo de alimentos son de baja calidad nutritiva y a menudo densamente calóricos, con elevados contenidos de azúcar, sal o grasas saturadas. Una revisión sistemática de la evidencia publicada en un período de 20 años (más 60 estudios), realizada por el Instituto de Medicina de los Estados Unidos, concluyó que la exposición de los niños a la publicidad televisiva de alimentos no nutritivos es un importante factor de riesgo que contribuye la obesidad infantil (22). En forma contraria, el análisis de una muestra representativa nacional, concluyó que un menor TDVTV se asocia con una mejor calidad de la alimentación en niños y adultos estadounidenses (23) Otra revisión sistemática encontró que el efecto de los anuncios sobre el consumo de alimentos es consistente en pre-escolares y escolares (24).

Los niños mexicanos destacan negativamente por el excesivoTDVTV y la cantidad de anuncios de alimentos no saludables que tienen que ver. Una revisión sistemática de los estudios realizados en más de medio siglo en diversos países encontró que el TDVTV en niños y adolescentes fue 2.4 horas por día (25). En México los niños de 8-14 años pasan en promedio cuatro horas diarias frente el televisor (26), es decir, $70 \%$ por sobre el promedio internacional. Se estima que un niño mexicano ve cada año alrededor de 12,700 anuncios de comida no saludable en televisión y ninguno de orientación nutricional, lo cual sitúa a México como el país que trasmite la mayor cantidad de anuncios de alimentos no saludables en todo el mundo dentro del horario infantil (27). Otro estudio realizado en niños de 6-13 años en San Luis Potosí, México, utilizando técnicas cualitativas y cuantitativas, mostró que un mayor TDVTV estaba asociado con un mayor consumo de alimentos no saludables, convirtiendo a la televisión es un mediador entre la obesidad y el consumismo (28).

Existen pocos estudios que han examinado el contenido nutricional de los productos anunciados en televisión, los cuales han encontrado que la mayoría de los alimentos anunciados son poco saludables, con altos contenidos en azúcar o grasa $(29,30)$. En México, un estudio encontró que los alimentos anunciados en programas infantiles tuvieron mayor contenido de calorías, grasa y carbohidratos (31).

El objetivo de este trabajo fue analizar el contenido nutricional de los alimentos anunciados por el Canal 5 de la empresa Televisa y la forma que puede asociarse con la obesidad en niños mexicanos. Se eligió este canal por ser el de mayor penetración en la audiencia infantil, ya que trasmite en señal abierta para el territorio nacional por medio de 67 estaciones repetidoras, además de que se incluye en todos los sistemas de cable y televisión satelital de paga. El Canal 5 Ilega a 24.6 millones de hogares, que representan $91.2 \%$ de los tele-hogares del país (32) y se estima que capta $68 \%$ de la audiencia de los niños de 4 a 12 años de edad (33).

\section{MATERIALES Y MÉTODOS}

Se utilizó un diseño no experimental descriptivo para analizar la publicidad dirigida a los niños mexicanos. Se grabaron los programas completos incluidos en la barra de programación infantil del Canal 5 de la empresa Televisa, durante la semana del 6 al 10 de febrero de 2012, de las 15:00-17:00 horas.

De manera independiente, cinco equipos de estudiantes de la Licenciatura en Nutrición Humana de la Universidad Autónoma Metropolitana, Unidad Xochimilco de la ciudad de México, cronometraron el tiempo de cada mensaje publicitario y en una base de datos en Microsoft Excel registraron los siguientes datos: duración del anuncio, producto o servicio anunciado y estrategia publicitaria utilizada. Se realizó una validación entre las cinco mediciones independientes para establecer la confiabilidad entre jueces y estandarizar los datos.

Posteriormente, se adquirieron los productos alimentarios anunciados, y a partir de la información de la etiqueta, se registró su contenido nutricional y los aditivos que contienen. En el caso de las comidas rápidas, se accedió a los sitios de internet mexicanos de las empresas, y cuando no fue posible obtener la información completa, se acudió a sus sitios web norteamericanos.

Se compararon los valores incluidos en la base de datos y, en caso de discrepancias, se cotejaron nuevamente las fuentes de información para obtener valores únicos. Finalmente, se agruparon los alimentos anunciados en función de su contenido de azúcares, grasa y sodio, tabulando los tiempos de trasmisión de cada tipo de alimento y grupo.

\section{RESULTADOS}

En promedio, en cada hora de programación se trasmitieron 12 minutos de anuncios y 48 minutos del programa trasmitido. El $36,4 \%$ del tiempo de los anuncios se utilizó para promover alimentos industrializados no saludables, que tienen bajo valor nutritivo y elevado contenido de azúcar, grasa o sodio. En contraste, no se trasmitió algún anuncio para promover el consumo de alimentos naturales o nutritivos ( $0 \%$ ). El 7,6\% fue ocupado por anuncios de juguetes; $9,1 \%$ por servicios gubernamentales; $3,8 \%$ por bancos y productos de higiene personal; y el restante $43,2 \%$ fue utilizado por Televisa para promover su propios programas, cuando estos espacios no han sido comercializados (figura 1).

En la semana analizada se trasmitieron 135 anuncios enfocados a promover el consumo de alimentos industrializados no saludables. El $74,0 \%$ del tiempo fue ocupado por productos con alto contenido de carbohidratos y azúcar; $17,5 \%$ por alimentos con alto contenido de grasa y sodio; y 8,5\% correspondió a lácteos con alto contenido de azúcar (tabla 1).

En la distribución del tiempo, en función de los grupos de productos, los cereales con azúcar ocuparon el primer sitio con $47,8 \%$ de la publicidad total de alimentos industrializados. Para llamar la atención de los niños, se utilizaron dibujos animados que promueven la aventura y diversión. Los cereales anunciados fueron Choco Crispis, Froot Loops y Zucaritas de la empresa Kellogg's, así como Trix y Cookie Crisp de la multinacional Nestlé.

El segundo grupo más anunciado fueron las papas fritas de la marca Sabritas, subsidiaria de PepsiCo, con 12,1\%, en donde actores o deportistas famosos promueven su consumo. El tercer sitio correspondió a las galletas Emperador de la empresa mexicana Gamesa, con 10,6\% del tiempo de anuncios. Esta empresa utilizó como incentivo el obsequio de 2 galletas adicionales en cada paquete de 6 galletas.

Los productos lácteos con alto contenido de azúcar ocuparon el cuarto sitio con el 8,5\%. En este grupo, los anuncios del producto Danonino de la empresa francesa Danone, sostienen que ayuda a aumentar la talla de los niños. El grupo de dulces 
y chocolates ocupó el quinto lugar con 8,3\%. Como ejemplo, el chocolate Carlos $V$ de la empresa Nestlé, que ofreció como incentivo la posibilidad de ganar premios sorpresa marcados en la parte oculta de las etiquetas.

En sexto lugar correspondió a los alimentos vendidos por las cadenas norteamericanas de comida rápida con 5,3\% de la publicidad de alimentos. Dentro de este grupo, la empresa McDonald's promovió su famosa "cajita feliz", así como el MacTrío, ambos paquetes integrados por hamburguesa, papas fritas y refresco. La "cajita feliz" es una caja de cartón, atractivamente diseñada y coloreada, que contiene un juguete o muñeco de regalo, además de los productos alimentarios. Este ha sido uno de los productos de mayor impacto en la historia de la compañía.

Las bebidas azucaradas, integrada por las norteamericanas Coca Cola y Tang, así como el refresco Boing, de la cooperativa mexicana Pascual, también ocuparon un $5.3 \%$ de la publicidad de alimentos.

Los cereales sin azúcar fue el grupo menos anunciado con $1,9 \%$. Los productos anunciados fueron el pan de caja de la empresa mexicana Grupo Bimbo, y las sopas instantáneas Maruchan de la empresa japonesa Toyo Suisan.

\section{FIGURA 1}

Porcentaje del tiempo de publicidad incluido en los programas infantiles del canal 5 de Televisa México, según tipo de anuncio ${ }^{1}$

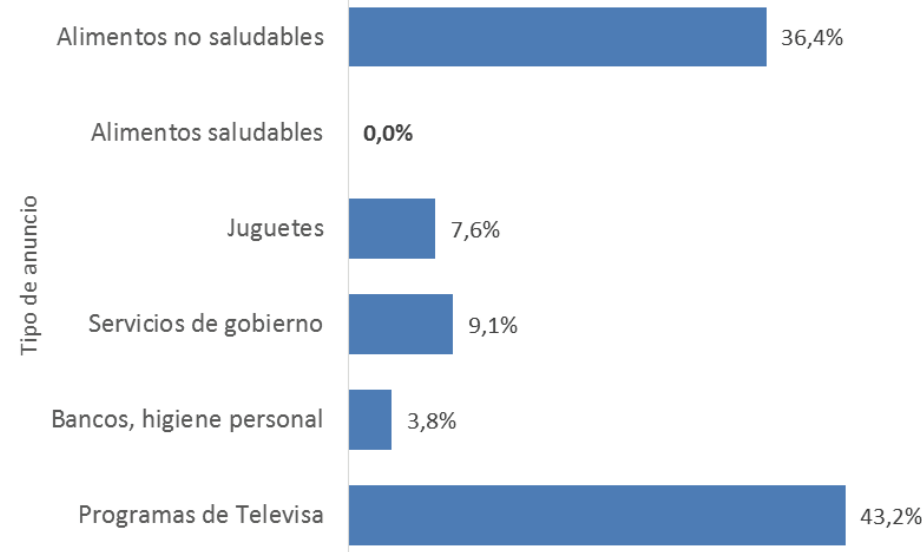

${ }^{1}$ Semana del 6 al 10 de febrero de 2012, de las 15:00-17:00 horas.

TABLA 1

Tiempo promedio y número de veces que se trasmitieron anuncios de alimentos industrializados no saludables en el canal 5 de Televisa México ${ }^{1}$

Tipo y grupo de alimento

$\begin{array}{cccc}\text { Tiempo } & \text { Número } & & \\ \text { promedio } & \text { de veces } & \text { Tiempo } & \% \text { del } \\ \text { del anuncio } & \text { que se } & \text { total (s) } & \text { tiempo total } \\ \text { (s) } & \text { transmitió } & & \end{array}$

Con alto contenido en azúcar

Cereales con azúcar (Choco Krispis, Froot Loops, Zucaritas, Trix y Cookie Crisp)

Galletas Emperador

Dulces y chocolates (Freskas, Bubulubu, Kinder sorpresa, Ice cubes)

Bebidas con azúcar añadida (Coca Cola, Tang, Boing)

Cereales sin azúcar (Pan Bimbo, Sopa Muruchan)

\begin{tabular}{lccc} 
& 90 & 1950 & $\mathbf{7 4 , 0}$ \\
30 & 42 & 1260 & 47,8 \\
20 & 14 & 280 & 10,6 \\
10 & 22 & 220 & 8,3 \\
20 & 7 & 140 & 5,3 \\
10 & 5 & 50 & 1,9 \\
\hline \multirow{2}{*}{20} & 30 & 460 & 17,5 \\
10 & 16 & 320 & 12,1 \\
& 14 & 140 & 5,3 \\
15 & 15 & 225 & $\mathbf{8 , 5}$ \\
- & 15 & 225 & 8,5 \\
\hline
\end{tabular}

Con alto contenido de grasa y sodio

Frituras (papas Sabritas)

Comida rápida (hamburguesas, pizzas, KFC, Subway)

Lácteos con alto contenido en azúcar

Lácteos con azúcar añadida (Danonino, yoghurt Alpura)

\section{Total}


El valor nutricional de los productos anunciados puede consultarse en la tabla 2.

DISCUSIÓN

La programación del canal 5 de la empresa Televisa de México promueve activamente el consumo de alimentos indus- trializados de alta densidad energética y con alto contenido de carbohidratos, azúcares, grasa y sodio.

Autores de otros países de América Latina han reportado situaciones semejantes. En Perú, un estudio mostró que los alimentos no saludables más publicitados en los programas más vistos, según el grupo de alimentos no saludables, fueron las

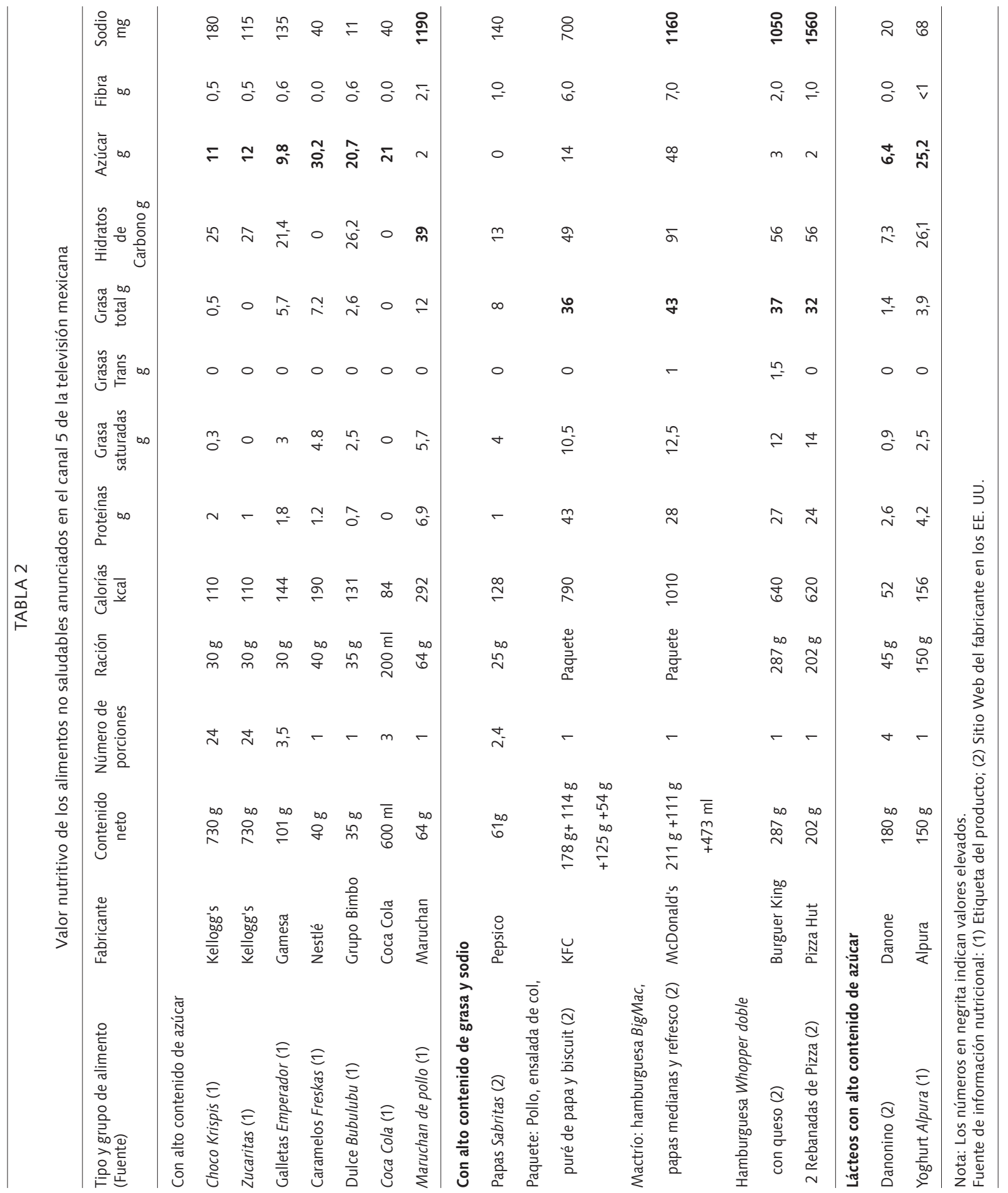


galletas $(31,5 \%)$, bebidas azucaradas $(30,4 \%)$, mientras que los de menor publicidad televisiva fueron los caramelos (9,2\%) (34). Un estudio chileno identificó que $73 \%$ de los alimentos anunciados en la televisión no son saludables por su alto contenido de azúcares y grasas (35). En México, otros estudios previos identificaron que los alimentos más anunciados eran las bebidas azucaradas, seguidos de los cereales industrializados con alto contenido de azúcar $(27,31)$. El presente estudio mostró que ese primer lugar corresponde a los cereales con azúcar, que son alimentos con mayor densidad energética.

La estrategia de promover el hábito de consumir cereales con alto contenido de azúcar en el desayuno de los niños o niñas en edad escolar es de alto riesgo. Al analizar el contenido nutricional de una ración de Choco Krispis, o de cualquier otro de los cereales anunciados, se estima que una ración de cereal con leche aportará 260 kcal (30 g de cereal más una tasa de leche entera) (36). Sin embargo, es frecuente que los niños consuman dos o tres raciones, lo que duplica o triplica el valor calórico a 520 y $780 \mathrm{kcal}$, respectivamente. Si el consumo adicional de una ración de cereal se convierte en un hábito, y además el escolar tiene una vida sedentaria y cubre sus requerimientos nutricionales con otros alimentos, 14 raciones extras de cereal repercutirán en una ganancia mensual de 500 a 1000 g, es decir, que en menos de un año se encontrará en el rubro de obesidad (37). Además sobrepasaría la recomendación dietética de proporcionar 20 a $30 \%$ de energía total, de las 1600-1800 kcal recomendadas para prevenir la obesidad en niñas y niños de 9 a 13 años, según la American Heart Association (AHA) (35) (tabla 3).

Por su parte, un paquete de galletas Emperador aporta 580 kcal, más un vaso de leche entera (200 ml, 150 kcal), sumaría un total de $730 \mathrm{kcal}$. Esta situación es similar al consumo de cereales con azúcar agregada, nada más que en este caso se anuncia como una "golosina", que puede acompañar al refrigerio escolar.

Si un niño en edad escolar desayuna una ración del cereal azucarado, más un refrigerio de galletas Emperador, el consumo de ambos productos representa un aporte total de $990 \mathrm{kcal}$; y si consume dos raciones de cereal y leche, los valores calóricos se incrementan a 1250-1510 kcal en tan sólo un tercio del día. En otras palabras, un consumo de esta naturaleza representa entre $78 \%$ y $94,3 \%$ del valor calórico de una dieta de 1600 kcal, cifra recomendada por la AHA (38), para niñas de 9 a 13 años (tabla 3). A los consumos anteriores, faltaría por sumar las calorías que aportarán los demás tiempos de comida, lo que seguramente conducirá a que el escolar sobrepase las recomendaciones de la ingesta calórica diaria, contribuyendo a un incremento diario en su peso corporal.. Además, debe considerarse que después del desayuno el niño entra en una etapa fisiológica de metabolismo en reposo al permanecer de 4 a 6 horas sentado en su aula escolar, lo que incrementa el riesgo de sobrepeso y obesidad.

Las hamburguesas son alimentos calóricamente elevados por el tamaño de las raciones y la cantidad de grasa que contienen. Las promociones invitan a que los consumidores agranden el tamaño de la hamburguesa o el refresco, sin considerar que en un vaso pequeño de refresco contiene $132 \mathrm{kcal}$, y si se opta por el tamaño mediano, el valor se incrementa a $380 \mathrm{kcal}$. La compra de estos paquetes no incluye el grupo de las frutas y verduras, además de que los mensajes publicitarios son promovidos por actores de moda de figura esbelta y de aspecto saludable.

El contenido de grasa y sodio es muy elevado en las hamburguesas promocionadas por Burguer King para los niños, ya que contienen $27 \mathrm{~g}$ de grasa de origen animal y $1050 \mathrm{mg}$ de sodio (tabla 2). La recomendación diaria de sodio para los grupos de población de 9 a 13 años, según la AHA, es menor a 2200 mg diarios, incluyendo el sodio contenido en forma natural de los alimentos y el que se utiliza en la preparación de los mismos. El consumo de un MacTrío McDonald's, representa más del 50\% del sodio recomendado en la dieta del niño. La grasa cubre $96 \%$ de la recomendación diaria en tan sólo una ración, además de que es grasa saturada. Si a ello se le agrega el sodio y la grasa del resto de los alimentos ingeridos en el día, así como las calorías de cereales, refrescos y otras golosinas, es muy probable que los niñas y niñas excedan las recomendaciones establecidas, incre-

TABLA 3

Recomendaciones de energía y raciones de grupos de alimentos para población por grupo de edad y sexo

\begin{tabular}{|c|c|c|c|c|c|}
\hline & 1 año & 2-3 años & 4-8 años & 9-13 años & 14-18 años \\
\hline Kilocalorías\& & 900 & 1000 & & & \\
\hline Mujeres & & & 1200 & 1600 & 1800 \\
\hline Hombres & & & 1400 & 1800 & 2200 \\
\hline$\%$ Grasa del total de kcal & $30-40$ & $30-35$ & $25-35$ & $25-35$ & $25-35$ \\
\hline Leche/día, tazas & 2 & 2 & 2 & 3 & 3 \\
\hline Carne/leguminosas, onzas & 1,5 & 2 & & 5 & \\
\hline Mujeres & & & 3 & & 5 \\
\hline Hombres & & & 4 & & 6 \\
\hline Frutas, tazas & 1 & 1 & 1,5 & 1,5 & \\
\hline Mujeres & & & & & 1,5 \\
\hline Hombres & & & & & 2 \\
\hline Vegetales, tazas & $3 / 4$ & 1 & & & \\
\hline Mujeres & & & 1 & 2 & 2,5 \\
\hline Hombres & & & 1,5 & 2,5 & 3 \\
\hline Cereales, onzas & 2 & 3 & & & \\
\hline Mujeres & & & 4 & 5 & 6 \\
\hline Hombres & & & 5 & 6 & 7 \\
\hline
\end{tabular}

\& Las calorías están estimadas en base a un estilo de vida sedentario y se deben aumentar de 0-200 kcal si es una actividad moderada y de 200-400 cuando es una actividad intensa.

Fuente: American Heart Association, Gidding SS, Dennison BA, Birch LL, Daniels SR, Gilman MW, et al. Dietary Recommendations for Children and Adolescents: A Guide for Practitioners. Pediatrics. 2006; 117(2):544-59. 
mentado el riesgo de padecer enfermedades cardiovasculares desde temprana edad.

Los alimentos tipo yogur de la marca Danonino y Alpura tienen un aporte considerable de azúcares simples de $6,4 \mathrm{~g}$ y 25,2 g, respectivamente. Además, están adicionados con colorantes (tartrazina) y saborizantes artificiales para simular el sabor y aroma de las frutas y utilizan mensajes engañosos para promover su consumo: el producto Danonino sostiene que su consumo ayuda a incrementar la talla del niño, lo cual es falso, ya que ésta se obtiene con una alimentación completa y saludable, no sólo consumiendo este tipo de yogurt. El valor nutritivo que aportan en proteínas es importante, sin embargo estas se pueden adquirir a un precio menor y sin necesidad de agregar azúcares extras a la dieta del niño.

Una reflexión final en este apartado nos lleva a preguntar: ¿cuántos segundos le bastan a la industria alimentaria y su publicidad para que los niños en México sean grandes consumidores de los productos no saludables? Además de que es muy probable que estos productos pueden impactar negativamente el estado de salud y nutrición de la población infantil de México, país que tiene una de las prevalencias más elevadas de sobrepeso y obesidad en el mundo.

La 63a Asamblea Mundial de la Salud, realizada en Nueva York en mayo de 2010, recomendó a los países miembros la necesidad de implantar políticas orientadas a reducir el impacto que tiene sobre los niños la promoción de alimentos ricos en grasas saturadas, ácidos grasos de tipo trans, azúcares libres o sodio. Para evitar la epidemia de la obesidad, los gobiernos deben trabajar de manera conjunta con las compañías productoras de alimentos industrializados para restringir la publicidad al público infantil de alimentos elevados en azúcar, sal y grasas; así como el promover alimentos saludables y fomentar la práctica de la actividad física (39).

El año 2008, once multinacionales, entre las que destacan Coca-Cola Company, Grupo Bimbo, Kellogg, McDonald's y Nestlé, agrupados en la International Food \& Beverage Alliance, se habían comprometido a impulsar iniciativas de publicidad responsable dirigida a los niños y restringir, de manera voluntaria, la publicidad de alimentos (40); sin embargo, el presente estudio mostró que no hubo restricción voluntaria en la publicidad dirigida a los niños de este tipo de alimentos.

Los niños y niñas expuestos a la televisión tienen mayor riesgo de presentar obesidad, porque desplazan la actividad física y se encuentran frente a un monitor que promueve el consumo de alimentos industrializados de alta densidad energética, mientras ven los programas infantiles. Este consumo, que puede ocasionar que los niños excedan sus requerimientos nutricionales, además la falta de actividad física ocasiona una disminución de su tasa metabólica por estar en reposo, puede incrementar el riesgo de obesidad. Ante esta situación la Academia Americana de Pediatría, recomienda limitar el uso de la televisión y/o videojuegos de 1 a 2 horas al día, y por cada hora adicional de TV el niño deberá realizar una actividad aeróbica adicional (38).

\section{CONCLUSIONES}

Los resultados del estudio muestran que la televisión mexicana realiza una promoción intensa de alimentos no saludables en la población infantil. Los mensajes publicitados por la TV mexicana buscan inducir a los niños a cambiar sus patrones alimentarios, al promocionar el consumo elevado de cereales con alto contenido de carbohidratos y azúcares simples; a la ingesta elevada de grasas saturadas y trans a través de la comida rápida; al bajo consumo de fibras (solubles e insolubles), de micronutrientes como el hierro, zinc, y potasio, así como de vitaminas A, D, C y el ácido fólico, ya que todos estos elementos están en baja cantidad en los productos analizados en este estudio. Aunque este estudio no tuvo el propósito de establecer una relación de causa-efecto entre la publicidad de alimentos no saludables y la obesidad infantil en México, los estudios realizados en otros países indican que la publicidad de alimentos dirigida a los niños constituye un factor determinante del estado nutricional y por lo tanto, que el excedente de energía que proporcionan los alimentos industrializados no saludables en la dieta, es muy probable que estén contribuyendo a las elevadas prevalencias de sobrepeso y obesidad en los niños y niñas mexicanas.

Por ello es urgente que el gobierno de México regule esta situación, ya que la auto-regulación publicitaria comprometida por las grandes trasnacionales de alimentos industrializados no ha funcionado en México. Las empresas multinacionales continúan invirtiendo cantidades multimillonarias en publicidad para manipular la demanda y los gustos de los niños hacia alimentos que pueden afectar su salud, al tiempo que promueven patrones alimentarios que contribuyen a elevar las principales conmoribilidades asociadas a la obesidad, como diabetes tipo 2, hipertensión arterial y dislipidemias. De no actuar con rapidez, será muy difícil comenzar a disminuir las elevadas prevalencias de sobrepeso y obesidad en México.

\section{RESUMEN}

El objetivo de este estudio fue analizar el contenido nutricional de los alimentos industrializados anunciados por el canal 5 de la televisión mexicana con mayor audiencia infantil y como puede asociarse con obesidad infantil. Se grabaron los programas de TV del 6 al 10 de febrero de 2012, en los horarios de mayor audiencia infantil. Cinco equipos independientes registraron y validaron la duración de cada anuncio, producto o servicio anunciado, la estrategia publicitaria, su contenido nutricional y los aditivos que contienen. Se trasmitieron 135 anuncios de alimentos industrializados no saludables. En contraste, no se trasmitió ninguno de alimentos naturales o nutritivos. El 74,0\% del tiempo fue ocupado por productos con alto contenido de carbohidratos y azúcar; $17,5 \%$ por alimentos con alto contenido de grasa y sodio; y 8,5\% correspondió a lácteos con alto contenido de azúcar. Los resultados muestran que la televisión mexicana promueve intensamente el consumo de alimentos no saludables en la población infantil. Es urgente que el gobierno de México regule la publicidad de alimentos no saludables en los horarios de programación infantil.

Palabras clave: niños; obesidad infantil; publicidad por TV; contenido nutricional; México.

\section{BIBLIOGRAFÍA}

1. World Health Organisation. Prioritizing areas for action in the field of population-based prevention of childhood obesity: a set of tools for Member States to determine and identify priority areas for action. Geneva, Switzerland: WHO; 2012.

2. Caballero B. The Global Epidemic of Obesity: An Overview. Epidemiologic Reviews. 2007;29(1):1-5.

3. World Health Organisation. Obesity and overweight (Fact sheet 311). Geneva: WHO;2012.

4. World Health Organization. Global status report on noncommunicable diseases 2010: Description of the global burden of NCDs, their risk factors and determinants. Italy: World Health Organization; 2011.

5. de Onis M, Blossner M, Borghi E. Global prevalence and trends of overweight and obesity among preschool children. Am J Clin Nutr. 2010;92(5):1257-64. 
6. Sassi F. Obesity and the economics of prevention : fit not fat. Northampton, MA: Edward Elgar Pub.; 2010.

7. OECD. Obesity update 2012. Paris, France: Organisation for Economic Co-operation and Development; 2012.

8. Gutiérrez JP, Rivera-Dommarco J, Shamah-Levy T, et al. Encuesta Nacional de Salud y Nutrición 2012: Resultados Nacionales. Cuernavaca, México: Instituto Nacional de Salud Pública; 2012.

9. Kirk SFL, Penney TL, McHugh TLF. Characterizing the obesogenic environment: the state of the evidence with directions for future research. Obesity Rev. 2010;11(2):109-17.

10. Swinburn B, Egger G, Raza F. Dissecting Obesogenic Environments: The Development and Application of a Framework for Identifying and Prioritizing Environmental Interventions for Obesity. Preventive Med. 1999;29(6):563-70.

11. Dietz Jr W, Gortmaker SL. Do we fatten our children at the television set? Obesity and television viewing in children and adolescents. Pediatrics. 1985;75(5):807-12.

12. Dennison BA, Erb TA, Jenkins PL. Television viewing and television in bedroom associated with overweight risk among low-income preschool children. Pediatrics. 2002;109(6):1028-35.

13. Gortmaker SL, Must A, Sobo IAM, Peterson K, Colditz GA, Dietz WH. Television Viewing as a Cause of Increasing Obesity Among Children in the United States, 1986-1990. Arch Pediatr Adolesc Med.. 1996;150(4):356-62.

14. Morales-Ruan MC, Hernandez-Prado B, Gomez-Acosta LM, Shamah-Levy T, Cuevas-Nasu L. Obesity, overweight, screen time and physical activity in Mexican adolescents. Salud Pública Mexico. 2009;51 Supp/ 4:S613-20.

15. Hernandez B, Gortmaker SL, Colditz GA, Peterson KE, Laird NM, Parra-Cabrera S. Association of obesity with physical activity, television programs and other forms of video viewing among children in Mexico city. Internat Journal of Obesity Related Metab Dis: J Internat Assoc Study Obes. 1999;23(8):845-54.

16. Dennison BA, Edmunds LS. The role of television in childhood obesity. Progress Pediatr Cardiol. 2008;25(2):191-7.

17. Hancox RJ, Milne BJ, Poulton R. Association between child and adolescent television viewing and adult health: a longitudinal birth cohort study. Lancet. 2004;364(9430):257-62.

18. Proctor MH, Moore $L L$, Gao D, et al. Television viewing and change in body fat from preschool to early adolescence: The Framingham Children's Study. Internat J Obes Related Metab Dis: J Internat Assoc Study Obesity. 2003;27(7):827-33.

19. Robinson TN. Television viewing and childhood obesity. Pediatric Clin North Am. 2001;48(4):1017-25.

20. Myers EF. ADA Evidence Analysis Library. J Am Dietetic Assoc. 2005;105(5 Supp/ 1):S79.

21. Zimmerman FJ, Bell JF. Associations of Television Content Type and Obesity in Children. Am J Public Health. 2010;100(2):334-340.

22. McGinnis JM, Gootman JA, Kraak VI. Food marketing to children and youth: threat or opportunity? Washington, D.C.: National Academies Press. Institute of Medicine (U.S.). Committee on Food Marketing and the Diets of Children and Youth.; 2006.

23. Sisson SB, Shay CM, Broyles ST, Leyva M. Televisionviewing time and dietary quality among u.s. Children and adults. Am J Prev Med. 2012;43(2):196-200.
24. Díaz Ramírez G, Souto-Gallardo MC, Bacardí Gascón M, Jiménez-Cruz A. Efecto de la publicidad de alimentos anunciados en la televisión sobre la preferencia y el consumo de alimentos: revisión sistemática. Nutr Hosp. 2011;26(6):1250-55.

25. Marshall SJ, Gorely T, Biddle SJH. A descriptive epidemiology of screen-based media use in youth: A review and critique. J Adoles. 2006;29(3):333-49.

26. Vazquez Mota J. Palabras de la Secretaria de Educación Pública, Josefina Vázquez Mota, durante la Sexta Asamblea General de la Asociación de Televisión Educativa Iberoamericana (ATEI): "Hacia la Televisión Iberoamericana del Conocimiento", realizada en el Centro Cultural Universitario, Tlatelolco. Sexta Asamblea General de la Asociación de Televisión Educativa Iberoamericana; 2008; México, DF. 24/11/2008.

27. Calvillo A. El ambiente obesigénico entre el poder legislativo y el ejecutivo: manifiesto por la salud alimentaria. México: El Poder del Consumidor;2007.

28. Moreno L. A, Toro Z. L. La televisión, mediadora entre consumismo y obesidad. Rev Chil Nutr. 2009;36(1):4652.

29. Taras HL, Gage M. Advertised foods on children's television. Arch Pediatr Adolescent Med. 1995;149(6):649-52.

30. Kotz K, Story M. Food advertisements during children's Saturday morning television programming: are they consistent with dietary recommendations? J Am Diet Assoc. 1994;94(11):1296-1300.

31. Perez-Salgado D, Rivera-Marquez JA, Ortiz-Hernandez L. Publicidad de alimentos en la programacion de la television mexicana: ¿los niños están más expuestos? Salud Pública Mexico. 2010;52(2):119-26.

32. Grupo Televisa. Reporte anual que se presenta de acuerdo con las disposiciones de carácter general aplicables a las emisoras de valores y a otros participantes del mercado de valores, por el año terminado el 31 de diciembre de 2012. México: Televisa;2013.

33. Grupo Televisa. Plan comercial 2012: televisa liderazgo. México: Televisa;2012.

34. Rojas-Huayllani EC, Delgado-Pérez DH. Influencia de la publicidad televisiva peruana en el consumo de alimentos no saludables en escolares de $4^{\circ}$ a $6^{\circ}$ primaria. An Fac Med. 2013;74(1):21-6.

35. Castillo Lancelloti C, Pérez Santiago O. Los anuncios de $T V$ dirigidos al público infantil promueven alimentos no saludables. Santiago de Chile: Centro de Estudios Liga Ciudadana de Consumidores; 2009.

36. Pérez de Gallo AB, Marván Laborde L. Dietas normales y terapéuticas: los alimentos en la salud y la enfermedad. México: La Prensa Médica Mexicana; 1996.

37. Williams SR. Manual práctico de Nutrición. México: Editorial Pax-México; 1983.

38. American Heart Association, Gidding SS, Dennison $B A$, et al. Dietary Recommendations for Children and Adolescents: A Guide for Practitioners. Pediatrics. 2006;117(2):544-59.

39. OMS. Conjunto de recomendaciones sobre la promoción de alimentos y bebidas no alcohólicas dirigida a los niños. Ginebra: Organización Mundial de la Salud; 2010.

40. IFBA. Five Commitments in Five Years: Review of Progress 2008 - 2013 Genève, Switzerland: International Food \& Beverage Alliance;2014. 